\title{
Zygomatico-Coronoid Ankylosis: A Case Report
}

\author{
Risimati Ephraim Rikhotso, Mbali Nkonyane \\ University of the Witwatersrand, Johannesburg, South Africa \\ Email: erikhotso@gmail.com
}

How to cite this paper: Rikhotso, R.E. and Nkonyane, M. (2017) Zygomatico-Coronoid Ankylosis: A Case Report. Open Journal of Stomatology, 7, 475-480. https://doi.org/10.4236/ojst.2017.711043

Received: September 4, 2017

Accepted: November 7, 2017

Published: November 10, 2017

Copyright (c) 2017 by authors and Scientific Research Publishing Inc. This work is licensed under the Creative Commons Attribution International License (CC BY 4.0).

http://creativecommons.org/licenses/by/4.0/

\begin{abstract}
Extra-articular ankylosis resulting from bony union between the coronoid process and the zygoma is very rare. It may result from facial fractures caused by gunshots, treated or untreated facial fractures or may arise as an extension of intracapsular ankylosis. We report a case of ankylosis between the coronoid process and the zygomatic arch in a 33-year-old Black male. The bony ankylosis was the result of a 2 year old untreated zygomatic arch fracture. Ankylosis developed secondary to heterotopic bone formation following trauma. The patient was treated by intra-oral coronoidectomy, followed by physiotherapy for three months. He returned for review one year later with a mouth-opening of $40 \mathrm{~mm}$ and a stable occlusion.
\end{abstract}

\section{Keywords}

Zygoma, Coronoid, Ankylosis, Extra-Articular

\section{Introduction}

Bony union between the coronoid process $(\mathrm{CP})$ and the zygoma is a very rare cause of extra-articular temporomandibular joint ankylosis [1]. It may result from facial fractures caused by gunshots, [2] treated or untreated fractures of the zygoma complex [3] [4] [5] with or without concomitant fractures of the CP, [6] chemical burns, mandibular fractures, [7] infection involving the infratemporal fossa, [8] local surgical complications [3] [9] [10] myositis ossificans [11] and extension of intracapsular ankylosis [10].

We report a case of ankylosis between the $\mathrm{CP}$ and the zygomatic bone in a 33-year-old Black male. This bony ankylosis was the result of an untreated zygomatic arch fracture sustained in a motor vehicle accident two years previously.

\section{Report of a Case}

A 33-year man presented to the maxillofacial and oral surgery outpatient clinic 
of the Chris Hani Baragwanath Academic Hospital (Johannesburg, South Africa) complaining of inability to open his mouth. He gave a history of being involved in a motor vehicle accident two years previously.

Patient's history indicated that following clinical and radiographic assessment then, he was diagnosed with an isolated left zygomatic arch fracture (Figure 1). He was admitted for surgery (Gillies lift) but absconded for fear of an operation. Since then the patient's mouth opening had progressively decreased.

Extra-oral examination revealed a depression over the zygomatic arch region. Interincisal mouth opening was zero and the patient could not perform any protrusive or lateral movements. Intra-oral examination however showed a stable occlusion and no further abnormalities.

Radiographic examination with 3-D recons CT scan, axial and coronal CTs revealed a bony mass bridging the $\mathrm{CP}$ and the zygomatic arch on the left side without capsular involvement (Figure 2 and Figure 3).

A diagnosis of zygomatico-coronoid ankylosis was made. The patient was taken to theatre and nasal intubation was performed fibre-optically. The left coronoid was exposed via an intra-oral incision. $\mathrm{CP}$ was confirmed to be fused with the ZA. A fissure bur was used to separate the left CP from the ramus (Figure 4).

The rest of the $\mathrm{CP}$ was separated from the inner aspect of the zygoma by means of an osteotome. The mass of bone and the $\mathrm{CP}$ attached to the zygoma were removed. The patient was then stretched to an interincisal mouth opening of $32 \mathrm{~mm}$. Patient commenced physiotherapy the following day and was discharged with an interincisal opening of $30 \mathrm{~mm}$. Figure 5 shows an orthopantomogram post-coronoidectomy. He returned 12 months later with an opening of $40 \mathrm{~mm}$ (Figure 6).

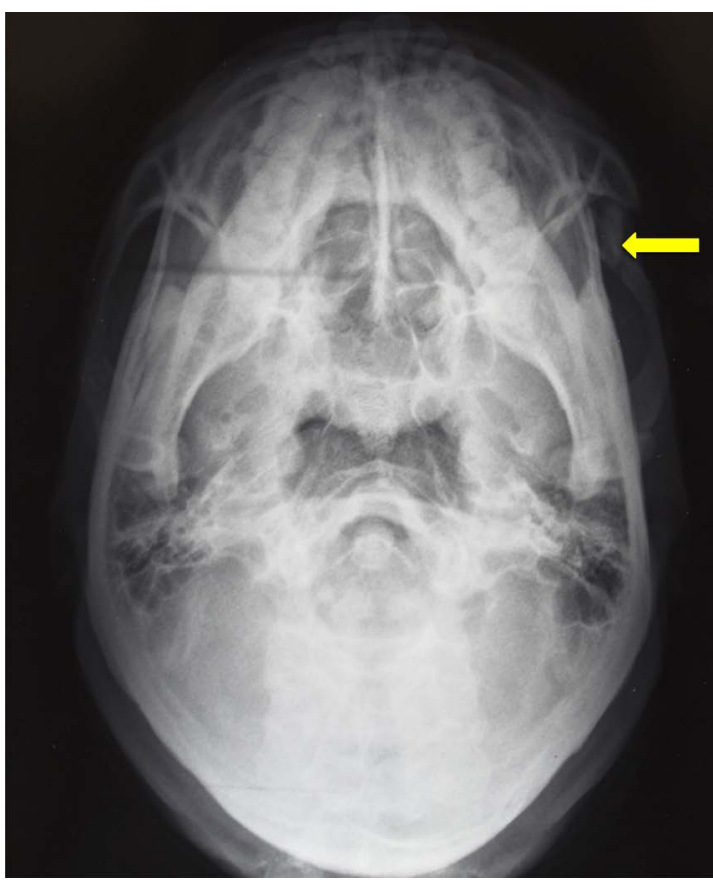

Figure 1. Submento-Vertex x-ray showing left zygomatic arch fracture (arrow). 


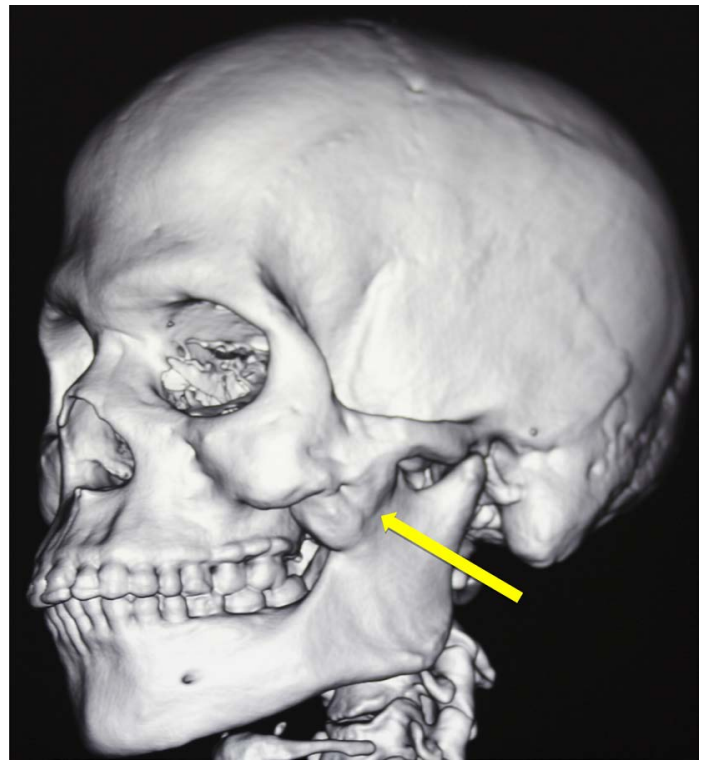

Figure 2. 3-D reconstruction illustrating bony mass between coronoid and zygoma (arrow).

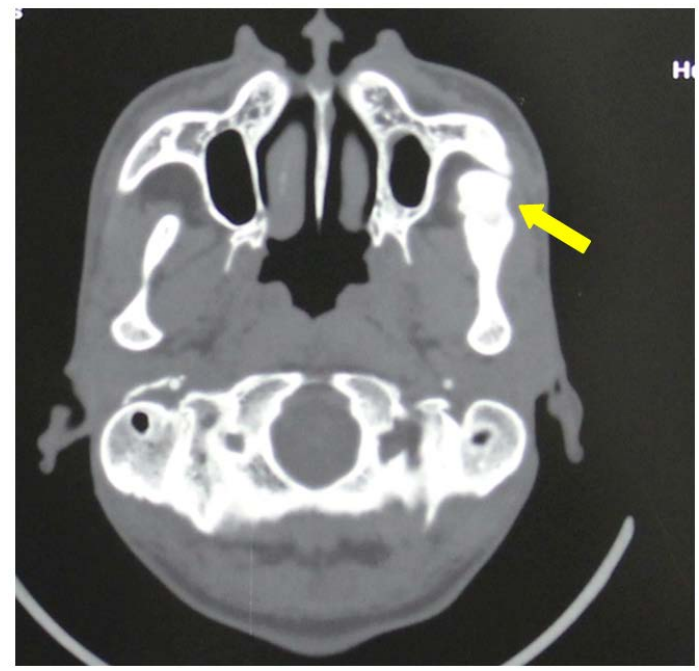

Figure 3. Axial CTs showing bony union between coronoid process and Zygoma (arrow).

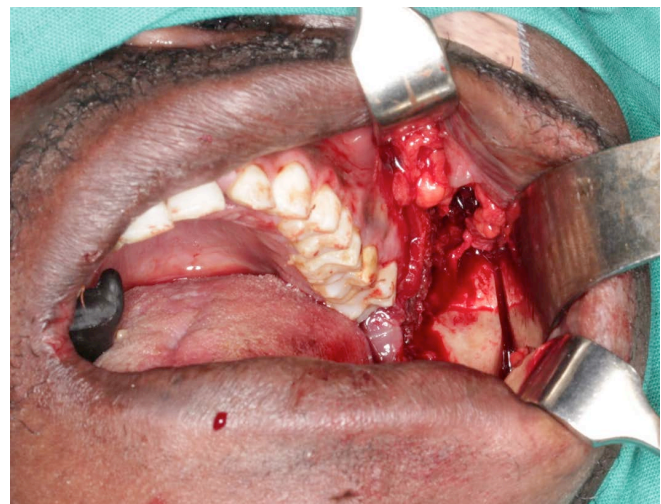

Figure 4. Intraoperative image showing osteotomy cuts. 


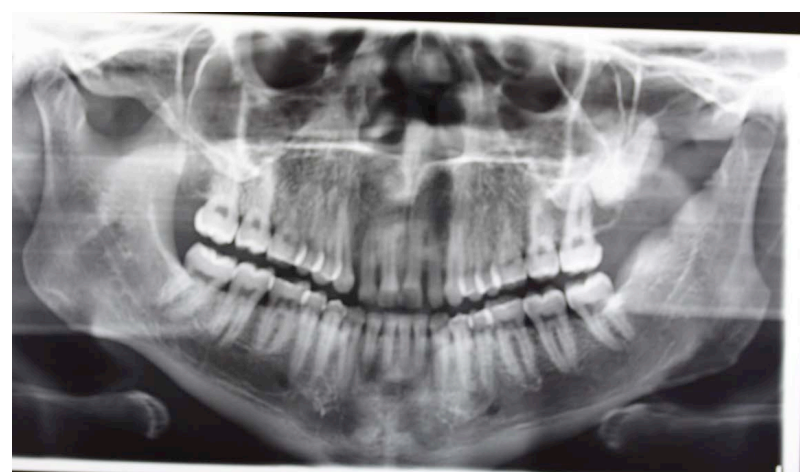

Figure 5. Post-operative orthopantomogram.

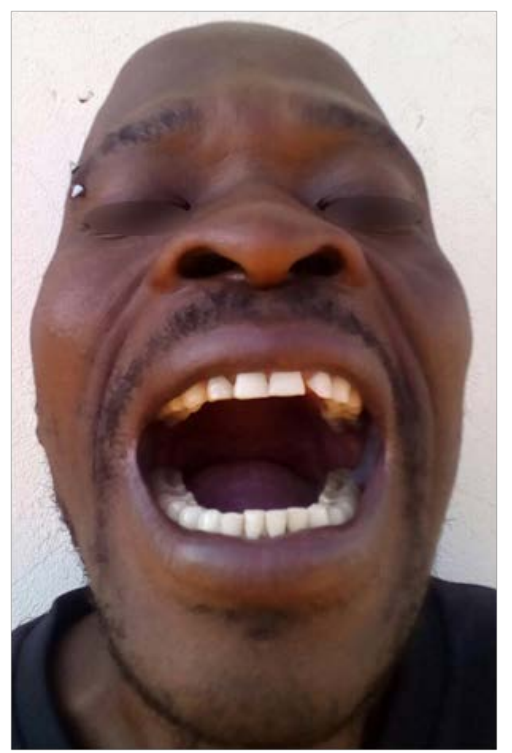

Figure 6. Inter-incisal mouth opening $(40 \mathrm{~mm}) 12$ months after the operation.

\section{Discussion}

Zygomatico-coronoid ankylosis is a very rare condition. This condition must be clearly distinguished from fibrous attachment of the ZA to the CP (zygomaticocoronoid fibrosis), a much more common clinical entity. Coronoidectomy is however the treatment of choice for both conditions [9].

Sequence of events that culminate in extra-articular TMJ ankylosis awaits elucidation, since heterotopic bone formation is rarely encountered in the maxillofacial region (examples of heterotopic bone formation in the maxillo-facial region include ossification of the stylohyoid ligament [12] periosteal ossification following subperiosteal haemorrhage or infection, and myositis ossificans of the masseter muscles [13]. It may results from metaplastic changes in CT elements that do not have osteogenic potential, following trauma, infection or surgery [1]. Histological examination generally reveals proliferating CT fibroblasts in transition to osteoblasts and areas of cartilage, osteoid and bone [9].

Most authors agree that the only possible treatment for extra-articular ankylosis is a coronoidectomy [1]. Diverse opinions as to whether the coronoid should 
be approached intra-orally or extra-orally exist. Extra-oral approach gives good access, but may result in a visible scar and CN VII palsy. Intra-orally there is no scar mark on the face and no risk of facial nerve injury, but access is very difficult.

In view of the large bony mass between the zygoma and the $\mathrm{CP}$, and limited mouth opening in our patient, the coronal approach seemed to be the best choice but the patient refused and instead opted for the intra-oral approach.

Early post-operative mouth opening exercises, a strict follow-up and even a stretch under general anaesthetic are imperative to prevent reankylosis. Some authors have stated that conventional procedures have shown a high rate of recurrence due to heterotopic bone and fibrous tissue formation, so they have used a coronoid osteotomy and insertion of a free abdominal flap. No fat graft was used in our patient.

Upon discharge, our patient was placed on an intense physiotherapy protocol, which was maintained for three months.

The patient was followed-up at six months and had a good mouth opening and function. He returned for review after one year with a mouth-opening of 40 $\mathrm{mm}$ and a stable occlusion.

\section{Conclusion}

Extracapsular temporomandibular bony ankylosis between the coronoid process and the zygomatic arch is a rare but noteworthy complication of zygoma fractures. We have presented such a case of fusion of the left coronoid process to the zygomatic bone in a 33-year-old male. The bony ankylosis was the result of a 2 year old untreated zygomatic arch fracture. Ankylosis developed secondary to heterotopic bone formation following trauma. The patient was treated by intraoral coronoidectomy. The rationale, indications and importance of post-operative physiotherapy are discussed.

\section{Conflict of Interest}

None.

\section{Compliance with Ethical Standards}

Informed consent was obtained from the patient.

\section{References}

[1] Agarwal, M., Gupta, D.K., Tiwari, A.D. and Jakhar, S.K. (2013) Extra-Articular Ankylosis after Zygoma Fracture: A Case Report and Review of Literature. Journal of Oral Biology and Craniofacial Research, 3, 105-107. https://doi.org/10.1016/j.jobcr.2013.05.003

[2] Brown, J.B. and Peterson, L.W. (1946) Ankylosis and Trismus Resulting from War Wounds Involving the Coronoid Region of the Mandible: Report of Case. Journal of Oral Surgery, 4, 258-266.

[3] Ostrofsky, M.K. and Lownie, J.E. (1977) Zygomatico-Coronoid Ankylosis. Journal 
of Oral Surgery, 35, 752-754.

[4] Rikalainen, R., Lamberg, M.A. and Tasanen, A. (1981) Extra-Articular Fibrous Ankylosis of the Mandible after Zygomatic Fracture. Journal of Maxillofacial Surgery, 9, 132-136. https://doi.org/10.1016/S0301-0503(81)80031-8

[5] Warson, R.W. (1971) Pseudoankylosis of the Mandible after a Fracture of the Zygomaticomaxillary Complex: Report of the Case. Journal of Oral Surgery, 29, 223-224.

[6] Marlette, R.H. (1963) Trismus and Pseudoankylosis Resulting from a Coronoid Zygomatico-Maxillary Fusion: Report of a Case. Journal of Oral Surgery, 21, 156-162.

[7] Allison, M.L., Wallace, W.R. and Von Wyl, H. (1669) Coronoid Abnormalities Causing Limitation of Mandibular Movement. Journal of Oral Surgery, 27, 229-233.

[8] Gridly, M.S. (1959) Abnormal Bony Connections between the Skull and Mandibles. Oral Surgery, Oral Medicine and Oral Pathology, 7, 954-962.

https://doi.org/10.1016/0030-4220(54)90293-5

[9] Schwartz, H.C. and Kagan, A.R. (1979) Zygomatico-Coronoid Ankylosis Secondary to Heterotropic Bone Formation: Combined Treatment by Surgery and Radiation Therapy: A Case Report. Journal of Maxillofacial Surgery, 7, 158-161. https://doi.org/10.1016/S0301-0503(79)80030-2

[10] Williams, A.C., Phillips, H., Rothman, B., Baurmash, H. and Matzkin, M. (1969) Ankylosis of the Coronoid Process to the Zygomatic Arch and Maxilla: Report of Case. Journal of Oral Surgery, 26, 804-806.

[11] Masaki, F., Takehiro, D., Ryuuichi, M. and Kumi. M. (2000) Release of Extra-Articular Ankylosis by Coronoidectomy and Insertion of a Free Abdominal Flap: Case Report. Journal of Cranio-Maxillo-Facial Surgery, 28, 369-372.

[12] Gossman, J.R. and Tarsitano, J.J. (1977) The Styloid Stylohyoid Syndrome. Journal of Oral Surgery, 35, 555.

[13] Plezia, R.A., Mintz, S.M. and Calligaro, P. (1977) Myositis Ossificans Traumatica of the Masseter Muscle. Oral surgery, Oral medicine and Oral Pathology, 44, 351. 